\title{
Erratum: On the classification of 6D SCFTs and generalized ADE orbifolds
}

\author{
Jonathan J. Heckman, ${ }^{a}$ David R. Morrison ${ }^{b, c}$ and Cumrun Vafa ${ }^{a}$ \\ a Jefferson Physical Laboratory, Harvard University, \\ Cambridge, MA 02138, U.S.A. \\ ${ }^{b}$ Department of Mathematics, University of California Santa Barbara, \\ CA 93106, U.S.A. \\ ${ }^{c}$ Department of Physics, University of California Santa Barbara, \\ CA 93106, U.S.A. \\ E-mail: jheckman@physics.harvard.edu, drm@physics.ucsb.edu, \\ vafa@physics.harvard.edu
}

ERRATUM TO: JHEP05(2014)028

ArXiv EPRINT: 1312.5746

\section{List of corrections:}

- In section 4, page 12, a characterization of D-type orbifold singularities associated with the configuration of curves $D\left(y \mid x_{1}, \ldots, x_{\ell}\right)$ is given. In this case, the group is actually a generalized D-type group, and is often labelled as $D_{p+q, q}$ where $p$ and $q$ are associated with the Hirzebruch-Jung continued fraction:

$$
\frac{p}{q}=(y-1)-\frac{1}{x_{1}-\ldots \frac{1}{x_{\ell}}} .
$$

For the present work the explicit form of this group is not needed. Similar considerations apply to the discussion in appendix D for D-type orbifold singularities. For further discussion see e.g., reference [1], as well as [2, 3].

- In section 5 , table 1 , there are a few typos in the list of endpoints at a small number of curves. The corrected entries are presented in the included table, and are explained more fully in the latest arXiv version of the present paper.

- In section 7, the proposed duality moves were revisited in reference [4] where it was found that the proposed duals have distinct anomaly polynomials. Therefore these theories are not duals, but are instead distinct. Similar considerations apply to the discussion in appendix E. This is explained more fully in the latest arXiv version of the present paper. 


\begin{tabular}{|c|c|c|c|c|c|c|c|c|c|}
\hline 1 & 2 & 3 & 4 & 5 & 6 & 7 & 8 & 9 & 10 \\
\hline \multicolumn{10}{|l|}{ (12) } \\
\hline & 92 & & & & & & & & \\
\hline & 83 & 822 & 8222 & 82222 & & & & & \\
\hline & 77 & 742 & 7322 & 73222 & 732222 & & & & \\
\hline & & 733 & 7233 & 72322 & 723222 & 7232222 & & & \\
\hline & & 727 & 7242 & 72242 & 722322 & 7223222 & 72232222 & 722232222 & 7222232222 \\
\hline & & 352 & 7227 & 72233 & 722242 & 7222322 & 72223222 & 722223222 & 7222223222 \\
\hline & & 343 & 3422 & 72227 & 722233 & 7222242 & 72222322 & 722222322 & 7222222322 \\
\hline & & 262 & 3342 & 34222 & 722227 & 7222233 & 72222242 & 722222242 & 7222222242 \\
\hline & & & 3333 & 33322 & 342222 & 7222227 & 72222233 & 722222233 & 7222222233 \\
\hline & & & 2522 & 33242 & 333222 & 3332222 & 72222227 & 722222227 & 7222222227 \\
\hline & & & 2442 & 33233 & 332322 & 3323222 & 33232222 & 332232222 & 3322232222 \\
\hline & & & & 2522 & 332242 & 3322322 & 33223222 & 332223222 & 3322223222 \\
\hline & & & & 24322 & 332233 & 3322242 & 33222322 & 332222322 & 3322222322 \\
\hline & & & & 24242 & 252222 & 3322233 & 33222242 & 332222242 & 3322222242 \\
\hline & & & & 22422 & 243222 & 2432222 & 33222233 & 332222233 & 3322222233 \\
\hline & & & & & 242322 & 2423222 & 24232222 & 242232222 & 2422232222 \\
\hline & & & & & 242242 & 2422322 & 24223222 & 242223222 & 2422223222 \\
\hline & & & & & 224222 & 2422242 & 24222322 & 242222322 & 2422222322 \\
\hline & & & & & 223322 & 2242222 & 24222242 & 242222242 & 2422222242 \\
\hline & & & & & & 2233222 & 22332222 & 223232222 & 2232232222 \\
\hline & & & & & & 2232322 & 22323222 & 223223222 & 2232223222 \\
\hline & & & & & & 2224222 & 22322322 & 223222322 & 2232222322 \\
\hline & & & & & & & 22242222 & 222332222 & 2223232222 \\
\hline & & & & & & & 22233222 & 222242222 & 2222332222 \\
\hline
\end{tabular}

Open Access. This article is distributed under the terms of the Creative Commons Attribution License (CC-BY 4.0), which permits any use, distribution and reproduction in any medium, provided the original author(s) and source are credited.

\section{References}

[1] O. Iyama and M. Wemyss, The classification of special Cohen-Macaulay modules, Math. Zeitsch. 265 (2009) 41 [arXiv:0809.1958].

[2] O. Riemenschneider, Deformationen von Quotientensingularitäten (nach zyklischen Gruppen), Math. Ann. 209 (1974) 211.

[3] E. Brieskorn, Rationale singularitäten komplexer flächen, Invent. Math. 4 (1968) 336.

[4] J.J. Heckman, D. R. Morrison, T. Rudelius and C. Vafa, Geometry of 6D RG flows, arXiv: 1505.00009. 